\title{
SITUAÇÃO PROBLEMA COMO ESTRATÉGIA DE ENSINO A DISTÂNCIA SOBRE PREVENÇÃO DE LESÃO POR PRESSÃO
}

Claudia Labriola de Medeiros Martins ${ }^{1} \quad$ https://orcid.org/0000-0002-0993-7348 Gisella de Carvalho Queluci ${ }^{1} \quad$ https://orcid.org/0000-0003-0496-8513

Objetivo: descrever o desenvolvimento de uma plataforma virtual de aprendizagem com resolução situacional de problemas como ferramenta inovadora de ensino de prevenção de lesão por pressão. Metodologia: Estudo metodológico, de abordagem descritiva, com estratégia pedagógica de resolução de situação problema segundo Vilma de Carvalho, desenvolvido em 06 etapas didáticas. Resultados: O delineamento metodológico adotado prevê o desenvolvimento das seguintes etapas: $1^{\mathfrak{a} \text { a }}$ etapa - apresentação do curso e resolução de pós-teste; $2^{a}$ a etapa - leitura de situação - problema e resolução de situações problemas; 3 â etapa - realização de pesquisa em bases de dados e fórum de discussão; 4ạ etapa - apresentação gráfica e audiovisual de conteúdo pedagógico; 5 ạ etapa - resolução de pós - teste; 6a etapa - avaliação do produto desenvolvido. Conclusão: A incorporação da metodologia de educação a distância e de resolução de situação problema são de extrema relevância ao desenvolvimento do raciocínio crítico - reflexivo na qualificação e atualização profissional em saúde.

Descritores: educação à distância; lesão por pressão; educação em enfermagem; aprendizagem baseada em problemas; enfermagem

\section{SITUATION RESOLUTION AS A DISTANCE TEACHING STRATEGY ON PRESSURE INJURY PREVENTION}

Objective: To describe the development of a virtual learning platform with situational problem solving as an innovative teaching tool for pressure injury prevention. Methodology: Methodological study, descriptive approach, with pedagogical strategy of problem solving according to Vilma de Carvalho, developed in 06 didactic steps. Results: The adopted methodological design foresees the development of the following steps: 1st stage - course presentation and post-test resolution; 2nd stage - situation reading - problem and problem solving: 3rd stage - conducting research in databases and discussion forum; 4th stage - graphic and audiovisual presentation of pedagogical content; 5th step - post test resolution; 6th step - evaluation of the developed product. Conclusion: The incorporation of the distance education methodology and problem situation resolution are extremely relevant to the development of critical - reflexive reasoning in the qualification and professional updating in health.

Descriptors: distance education; pressure injury; education in nursing; problem-based learning; nursing

\section{SITUACIÓN RESOLUCIÓN COMO ESTRATEGIA DE ENSEÑANZAA DISTANCIA SOBRE PREVENCIÓN DE LESIONES POR PRESIÓN}

Objetivo: describir el desarrollo de una plataforma virtual de aprendizaje con resolución de problemas situacionales como una herramienta de enseñanza innovadora para la prevención de lesiones por presión. Metodología: Estudio metodológico, enfoque descriptivo, con estrategia pedagógica de resolución de problemas según Vilma de Carvalho, desarrollado en 06 pasos didácticos. Resultados: El diseño metodológico adoptado prevé el desarrollo de los siguientes pasos: la etapa: presentación del curso y resolución posterior a la prueba; 2da etapa - lectura de la situación - problema y resolución de problemas; 3a etapa: investigación en bases de datos y foro de discusión; 4a etapa: presentación gráfica y audiovisual de contenido pedagógico; 5to paso - resolución posterior a la prueba; Sexto paso: evaluación del producto desarrollado. Conclusión: La incorporación de la metodología de educación a distancia y la resolución de situaciones problemáticas son extremadamente relevantes para el desarrollo del razonamiento crítico - reflexivo en la calificación y actualización profesional en salud.

Descriptores: Educación a Distancia; Úlcera por Presión; Educación en Enfermería; Aprendizaje Basado en Problemas; Enfermería.

${ }^{1}$ Escola de Enfermagem Aurora de Afonso Costa. Universidade Federal Fluminense, RJ

Autor Correspondente: Claudia Labriola de Medeiros Martins - E-mail: claudialabriola@gmail.com 


\section{INTRODUÇÃO}

As lesões por pressão são a terceira condição mais cara após o câncer e as doenças cardiovasculares. Os profissionais de enfermagem são responsáveis pelo cuidado direto e contínuo, além do tratamento e prevenção. Para alcançar um atendimento de qualidade ideal, os conhecimentos e atitudes de enfermagem devem ser baseados nas melhores evidências científicas ${ }^{1}$.

A incidência e a prevalência de lesões por pressão nos cenários assistenciais são variáveis ${ }^{2}$, no qual identifica-se, nos Estados Unidos, em pacientes críticos, prevalência de $8,8 \%$ a $9,3 \%^{3}$. Em contrapartida no Brasil, essas taxas em idosos internados correspondem a $18,8 \%$ e, em pacientes de unidades de terapia intensiva, corresponde a $17,7 \%$ a $35,2 \%{ }^{2}$.

Estudos internacionais apontam uma incidência entre $4,5 \%$ a $25,2 \%$ (Reino Unido), e uma prevalência de 2,9\% a 8,34\% (Espanha), 18,4\% (Inglaterra) e 19,1\% (Estados Unidos $)^{4-5}$. No Brasil existem poucos estudos sobre incidência do agravo, no entanto alguns estudos existentes apontam entre $41,2 \%$ e $59 \%$ de risco para o desenvolvimento da lesão por pressão, e uma prevalência entre $8 \%$ e $23 \%$.

Nesse sentido, a assistência de enfermagem à pessoa com ferida requer uma prática consistente e competência trabalhada ao longo da graduação, não se extinguindo após sua conclusão, tendo em vista a atenção à saúde e a importância das tecnologias educacionais na maior efetividade do cuidado ${ }^{7}$.

Com isso, surge a possibilidade das metodologias ativas de ensino, como importante estímulo à aprendizagem e promoção do pensamento crítico-reflexivo, à motivação, criatividade, integração do conhecimento e habilidades interpessoais ${ }^{8}$. A metodologia de resolução de problemas torna-se importante nesse sentido, na medida em que se apoia em uma abordagem situacional por meio do encontro do profissional de enfermagem com as situações dos clientes, efetivando um processo de ensino e aprendizagem crítico.

Deste modo, a partir de tais considerações, tem - se por objetivo descrever o desenvolvimento uma plataforma virtual de aprendizagem com resolução situacional de problemas para enfermeiros e acadêmicos de enfermagem como ferramenta inovadora de ensino de prevenção de lesão por pressão.

\section{METODOLOGIA}

\section{Tipo de Estudo}

Estudo metodológico, do tipo discursivo e abordagem qualitativa, com estratégia pedagógica de resolução de situações - problema segundo Vilma de Carvalho, que visa promover um processo de reflexão acerca da prática profissional e da teoria, de forma conjunta e inovadora.

\section{Local do estudo}

O presente estudo integra as investigações desenvolvidas pelo Mestrado Profissional de Ensino da Saúde (MPES), na linha de pesquisa de formação pedagógica em saúde, durante o período de julho a agosto de 2019.

\section{Coleta dos dados}

Dados extraídos da Dissertação de Mestrado “Tecnologia Virtual de Aprendizagem: Curso à Distância de Situação - Problema Como Estratégia de Ensino Para Prevenção de Lesão Por Pressão", apresentada à Escola de Enfermagem Aurora de Afonso Costa, Universidade Federal Fluminense, Niterói, RJ, Brasil.

\section{Procedimentos de análise dos dados}

Os dados foram analisados de forma discursiva através da descrição das etapas pedagógicas.

\section{Procedimentos éticos}

Este estudo foi submetido e aprovado pelo Comitê de Ética e Pesquisa, de acordo com parecer no: 3.375 .082 e certificado de apresentação para apreciação ética (CAEE) no 12129719.1.0000.524.

\section{RESULTADOS}

\section{Desenvolvimento da Plataforma Virtual de Aprendizagem}

O ambiente virtual de aprendizado (AVA) produzido neste estudo desenvolveu-se por meio da plataforma denominada Modular Object-Oriented Dynamic Learning Environment (MOODLE), que é um ambiente virtual de aprendizagem a distância, considerado um software livre, devido a sua gratuidade.

Dessa forma, para desenvolvimento da plataforma virtual de aprendizagem com estratégia pedagógica resolução de situação-problema foram realizados dois encontros com a coordenação do ensino a distância (CEAD) da instituição de estudo. No primeiro encontro foi discutido a proposta do curso e detalhamento das etapas a serem desenvolvidas e no segundo encontro ocorreu a capacitação para manipular a plataforma e construção do produto.

O curso possuiu data de início e término e, antes de iniciar disponibilizou - se uma mensagem de boas-vindas e uma breve explicação sobre as etapas a serem seguidas. O aluno só consegue avançar para etapa seguinte quando concluir a etapa anterior. Na figura l é possível observar a descrição detalhada das etapas pedagógicas do curso. 
Figura 1 - Esquema ilustrativo de construção das etapas pedagógicas do curso, Niterói, 2019.
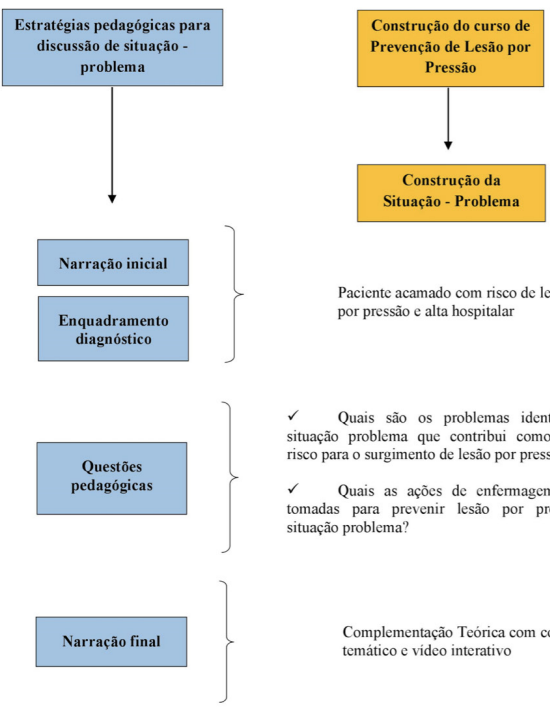

Paciente acamado com risco de lesão por pressão e alta hospitalar

Quais são os problemas identificados na situação problema que contribui como fatores de $\checkmark$ Quais as ações de enfermagem dever ser tomadas para prevenir lesão por pressão nesta
situação problema?

Complementação Teórica com conteúdo temático e video interativo

Fonte: Martins e Queluci, 2019

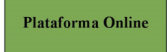

Mensagem
de Boas Vindas

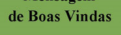

Pré Teste

Leitura da

Identificarõo do Problemas

Sugestão de Ações de Prevenção

Pesquisa Online

Fórum de Discussão
Pós Teste e Avaliação da Plataforma e todas as etapas do curso

\section{Estratégia Pedagógica de Resolução de Situação Problema}

A metodologia de resolução de situações-problema é uma importante alternativa metodológica desenvolvida pela Professora Dra. Vilma de Carvalho, durante a inserção de novas metodologias ao Curso de Graduação da Escola de Enfermagem Anna Nery, da Universidade Federal do Rio de Janeiro (EEAN/UFRJ) $)^{8-9}$

Buscando desenvolver um perfil profissional, esta metodologia embasa - se em premissas importantes que norteiam a construção da formação, por meio da ideia de integração entre a teoria e a prática, visando contextualizar o estudo com o trabalho, no qual o centro de interesse do ensino é deslocado para as atividades do estudante, sendo ele o agente do próprio aprendizado, mediante a situações e experiências cotidianas?.

Para isso, aponta-se a necessidade de investimentos pedagógicos, que possam contribuir pela conjunção do "saber fazer" crítico-reflexivo e do "poder fazer" na prática assistencial da realidade daqueles que receberão o atendimento profissional ${ }^{10}$

Dessa forma, “a estratégia de resolução de situações problema são, em sua maioria, emergentes e adotadas na base do consenso coletivo"9:164, haja visto que, objetiva - se reorientar a aprendizagem quanto à formação da consciência crítica, possibilitando o exercício da criatividade, do julgamento, das decisões e também das intervenções do profissional da enfermagem.

A sequência das etapas pedagógicas é representada pelos “Diagnósticos da situação de saúde", "Planejamento das Intervenções para a Resolução dos Problemas Identificados", "Execução das Intervenções" e "Avaliação da Ajuda Prestada".

É indispensável, portanto, uma formação em Enfermagem que direciona o desenvolvimento profissional em relação as "ações competentes e capazes de se explicar em bases científicas, e em relação ao saber-fazer e ao poder-fazer, as coisas de seu interesse e sua responsabilidade"10:117.

Assim, mediante esse contexto, o ensino de Enfermagem deve preparar e legitimar os individuos para este trabalho, "através do aparato técnico-científico e ético-filosófico e do encontro com as situações dos clientes" $8: 15$, tendo na metodologia de resolução de problemas a viabilização dos elementos fundamentais para a formação profissional em detrimento das demais, proporcionando "a formação da consciência crítica e a aquisição de competências" que se adequam às mudanças e às demandas presentes no campo da saúde $9: 162$.

\section{Construção da Situação-Problema}

Para a elaboração da situação-problema de cliente com riscos de lesão por pressão, foram utilizadas fontes da literatura, como livros, artigos, revistas, e elementos pertinentes da práxis da pesquisadora. Ressalta-se que tal situação é de natureza hipotética, porém realística, sendo constituídas de problemas possiveis do mundo real na prática profissional do enfermeiro. Para isso, é importante que os conteúdos em abordagem sejam autênticos, relevantes e representativos daquilo que se espera na prática cotidiana do profissional.

Nesse sentido, considera - se que "Devem ser evitadas informações sem propósito, que acabam tirando o foco do problema". Assim, o contexto deve ser adequado à prática, de forma realista, abordando casos e causas, propondo de forma contextualizada, reflexões e discussões que possam favorecer a identificação total do problema, a fim de que se possa planejar as soluções técnicas e procedimentais corretas para solucionar o problema do paciente. Caso contrário, se o problema se apresenta de forma muito artificial, “(...) a discussão ficará no questionamento de hipóteses da situação fictícia, gerando um resultado frustrante"11:150

Durante o processo de geração da situação-problema, deve ser levado em consideração a importância da criatividade, da capacidade de explorar e refletir, do raciocínio sensivel e intuitivo do pesquisador. Além do mais, destaca - se que "a necessidade de serem evitados prejuízos de valores por parte do pesquisador durante o desenvolvimento das questões dos clientes a serem problematizados a fim de que se produza apoio dos princípios éticos, e não abordagens moralistas (...)"8:45 
Com isso, para o conteúdo das situações desenvolvidas, foram considerados os aspectos que são percebidos ou visualizados como sendo alguns "desvios" da ação profissional ou que "desvirtuam" a função do enfermeiro. Esses aspectos, presentes no roteiro para a elaboração da situação problema foram, então, elencados de acordo com a sua pertinência no que diz respeito aos cuidados de Enfermagem para com o cliente.

Neste contexto, é importante considerar que o cuidado de Enfermagem e o ensino deste nem sempre são "apropriadamente discutidos em âmbito de temática de situações de enfermagem; não nas circunstâncias problemáticas do cotidiano do cuidar de pacientes ou dos posicionamentos dilemáticos em face dos atendimentos executados pelos exercentes e em nome do agir da profissão"10:260.

Essa construção envolve a "Abordagem Inicial à Situação", aplicada como experiência de aprendizagem que representa o que o enfermeiro encontra em sua prática de atuar e cuidar dos pacientes.

Baseado na situação-problema em descrição, foram elaboradas perguntas problematizadoras, tendo como referência o “Levantamento de Questões Pedagógicas". Estas objetivaram provocar o questionamento, estimular o pensamento crítico-reflexivo do estudante e servir como um gatilho para busca por informações relevantes para a identificação dos problemas os quais os pacientes apresentam em relação à lesão por pressão.

As perguntas pedagógicas serviram de norte para conduzir os participantes aos próximos passos, os quais são contemplados na "Narração Final". Nesta etapa, foram descritos os problemas e sua discussão, sob a visão da Enfermagem, com vistas à resolução da situação-problema do cliente. Para isso, a pesquisa seguiu com a apresentação dos problemas do paciente com riscos de lesão por pressão e, posteriormente, a análise de possiveis soluções.

\section{ETAPAS PEDAGÓGICAS}

\section{Etapa 01: Pré Teste}

O pré-teste contém vinte questões objetivas, com opções de verdadeiro ou falso, sendo possivel apenas uma tentativa de resolução, cujo resultado é disponibilizado através de nota (de 0 a 20 pontos).

Após a realização do pré-teste, os participantes passam para a próxima etapa do curso, que consiste na leitura da situação-problema sobre um paciente com risco de lesão por pressão e que recebeu alta hospitalar. Mediante a esta problemática, são considerados elementos de um roteiro com questões norteadoras acerca das interferências, acometimentos e diversas necessidades deste paciente.

\section{Etapa 02: Leitura da Situação Problema e Resolução das Perguntas Pedagógicas}

Mediante a leitura da situação - problema, os participantes identificam os principais problemas da situação apresentada. Para isso, também apresentou - se uma mensagem com a explicação da realização desta, para esclarecimento de dúvidas sobre as questões e indicações de materiais de pesquisas, que o auxiliariam na resolução das questões e conteúdo desta segunda etapa.

O objetivo desta etapa é que o participante seja estimulado a buscar e identificar os problemas que podem estar inseridos no contexto apresentado de um paciente com riscos de lesão por pressão, quais riscos existentes e procedimentos adequados de prevenção. Além disso, devem estimular o pensamento crítico e multifacial acerca do problema em questão, proporcionando melhor direcionamento pedagógico.

As questões pedagógicas valem 10 pontos cada, totalizando 20 pontos a nota máxima, tendo que anexar na questão $n$ ㅇ 2, as referências utilizadas. É importante, portanto, compreender as características e os cuidados necessários em relação ao paciente idoso do sexo masculino com hiperemia em região sacra em estágio I. Foram perguntas pedagógicas deste estudo:

1) Quais os problemas identificados na situação - problema?

2) Quais as ações de enfermagem devem ser tomadas para prevenir lesão por pressão nesta situação problema?

\section{Etapa 03: Realização de Pesquisa em Bases de Dados e Fórum de discussão}

O fórum de discussão ocorreu após a realização das pesquisas por parte dos participantes, servindo para elucidar o tema e avaliar os trabalhos apresentados, como uma forma de verificar o nível de entendimento em relação ao tema proposto.

Além disso, foi possivel melhorar a orientação e abordagem, sendo capaz de minimizar as dúvidas apresentadas mediante complementação teórica. A participação no fórum possuiu pontuação máxima de 40 pontos.

Esta etapa visa discutir uma situação - problema sobre um paciente com risco de lesão por pressão, abordando o que deve ser observado, como seria o plano de enfermagem para um eventual problema para com o paciente, quais as orientações e práticas a serem adotadas, dentre outras questões de relevância aos cuidados de enfermagem.

\section{Etapa 04: Conteúdo Programático}

Nesta etapa foi disponibilizado uma apresentação gráfica do tipo power point e uma mídia audiovisual cujo conteúdo re- 
fere - se a temática de prevenção de lesão por pressão. Estas, foram desenvolvidas com base em literaturas científicas, artigos, protocolos atualizados, contendo os seguintes tópicos:

- Conceitos Básicos de Lesão por pressão (Anatomia da pele, fisiopatologia, definição, estágios da LPP, regiões vulneráveis para formação de LPP;

- Escala de Braden;

- Grupos de riscos de LPP;

- Fatores Predisponentes a LPP;

- Complicações da LPP;

- Vídeo Animado com os pontos principais do Protocolo de prevenção de lesão por pressão da instituição.

\section{5a Etapa - Pós teste}

O pós-teste contou com 20 questões para marcar verdadeiro ou falso, sendo as mesmas perguntas do pré-teste, com uma tentativa para sua realização e nota de 0 a 20 pontos. O comprimento de todas as etapas do curso, totalizou máximo de 100 pontos.

A realização do pós-teste consiste em avaliar a aquisição de conhecimentos que os participantes obtiveram ao longo do curso, com questões básicas e fundamentais inclusas ao contexto trabalhado ao longo do curso.

\section{6a etapa - Avaliação do produto}

Nesta etapa realizou - se avaliação do curso e de todas as suas etapas, mediante questionário de avaliação disponível na plataforma moodle contendo seis questões.

Após a conclusão de todas as etapas, foi disponibilizado uma mensagem de agradecimento pela participação, contendo os nomes da pesquisadora, orientadora e da coordenação da educação a distância.

\section{DISCUSSÃO}

A modalidade de ensino a distância vem sendo amplamente discutida ao longo dos anos, geralmente compreendida como sendo uma possibilidade de ampliação ao acesso à cursos de qualificação profissional, muito comum no âmbito do ensino superior. Além disso é vista por muitos como uma possibilidade complementar ao ensino presencial, já que a mesma pode também ser feita completamente a distância ${ }^{12-13}$.

Ao fortalecer as competências individuais e coletivas dos educandos, correlacionando problematizações, adversidades e rotinas que subsidiam a prática profissional, proporcionando autonomia e coletividade, se favorece, o pensamento crítico, a criatividade e a iniciativa ${ }^{14}$.

Dessa forma, ressalta-se a extrema relevância e inovação do produto produzido por meio deste estudo, haja visto que, intervenções educativas como a metodologia de resolução de situação problema, enriquecem os modernos métodos pedagógicos, oportunizando experiências, conhecimentos e práticas singulares, articuladas a pluralidade dos cenários ${ }^{2,15}$.

Estudos apontam que o pensamento crítico reflexivo acerca das demandas e complexidades individuais do paciente promove o desenvolvimento de uma avaliação profunda e harmônica, e consequente reestabelecimento do bem-estar e bem viver ${ }^{15}$. Quanto aos desafios que emergem neste cenário, aborda - se a necessidade de adequação dos conteúdos online e os objetos de aprendizagem, a adequação das atividades propostas à realidade cursista e o desenvolvimento de competências tecnológicas ${ }^{16}$

Considerando essas questões, é importante inserir o profissional de saúde nesse contexto, onde o mesmo enfrenta uma série de desafios no que diz respeito a manutenção de sua atualização no ramo de atuação. $O$ intenso dia a dia da profissão impõe algumas dificuldades ao profissional da enfermagem, e geralmente esses desafios estão atrelados ao tempo, onde o mesmo encontra dificuldades para realizar cursos de aperfeiçoamento e atualização na sua área de atuação, que é imprescindível para que o profissional possa manter a sua prática com segurança e qualidade ${ }^{17}$.

Portanto, é de extrema relevância o desenvolvimento de metodologias que visam extrair dos alunos as suas próprias experiências, mediante também a um processo de reflexão acerca da prática e da teoria, onde de forma conjunta buscam soluções para a situação problema que the é apresentada. Assim, as "estratégias para a resolução de situações-problema são, em sua maioria, emergentes e adotadas na base do consenso coletivo"10:164

Assim, faz-se importante a compreensão situacional dos problemas de saúde e enfermagem, segundo sua gravidade, para que os estudos e práticas de ensinar, cuidar e aprender sejam intensificados ${ }^{15}$, haja vista sua significativa relevância ao planejamento e dimensionamento dos trabalhos e cuidados de enfermagem.

\section{Limitações do Estudo}

Este estudo apresenta limitações oriundas da avaliação pontual das variáveis investigadas, podendo configurar como obstáculo à generalização dos resultados, porém, traz avanços para a enfermagem em diversos campos, haja visto que os cursos de EAD são de extrema relevância para a qualificação e atualização profissional, na complementação teórica da graduação e pós-graduação em saúde.

\section{Contribuições do Estudo Para a Prática de Enfermagem}

Neste sentido, sobre as contribuições, destacam-se a re- 
dução de tempo e custos, a flexibilidade e a democratização do acesso e a capacidade territorial. Logo, importa discutir a formação e as demandas, bem como os métodos e as ferramentas adequadas para favorecer um ensino-aprendizagem de qualidade, haja visto que, no contexto da enfermagem, a avaliação realizada por aqueles que estão envolvidos nestas experiências com EaD, tem revelado contribuições e desafios ${ }^{21-22}$.

\section{CONCLUSÃO}

A construção de uma prática de ensino que seja de fato construtiva precisa ser pautada em pilares essenciais. No caso da modalidade de educação a distância é fundamental o preparo profissional, a estrutura de ensino e também a metodologia de trabalho, que foque no aluno como sendo o construtor do seu conhecimento, por meio de estímulos que possam desafia-lo a resolver refletir, resolver problemas e propor soluções. Além disso, é preciso estar inserido em um contexto próximo a prática profissional.

Considerando a importância de se ampliar o conhecimento nos variados níveis de formação frente a prevenção de lesões por pressão, entende-se que esta modalidade de ensino consolida a prática profissional em pilares essenciais, devendo assim, ser considerada como relevante subsídio a melhoria da qualidade da assistência e segurança em saúde, além de fortalecimento do Sistema Único de Saúde (SUS) e das Políticas Públicas.

Portanto, é imprescindivel o desenvolvimento de estratégias de ensino-aprendizagem, que possam ser aplicadas e utilizadas na educação em enfermagem, para que haja oportunização de conhecimentos e conteúdo específicos, aperfeiçoamento das competências, habilidades, práticas e condutas relacionadas à prevenção de lesões por pressão, sem comprometer a rotina de trabalho e estudo, além de produzir subsídios científicos, baseados em evidências, de impacto positivos e significativos ao panorama assistencial.

\section{Contribuição dos autores}

CLMM: Redação do artigo; Análise e interpretação dos dados.GCO: Concepção e delineamento; Revisão crítica; Aprovação da versão a ser publicada.

Agradecimentos: Ao Conselho Federal de Enfermagem

\section{REFERÊNCIAS}

1. Tirgari B, Mirshekari L, Forouzi M. A. Pressure Injury Prevention: Knowledge and Attitudes of Iranian Intensive Care Nurses. Adv Skin Wound Care. 2018 Apr; 31(4):1-8.

2. Caldini LN, Araújo TM, Frota NM, Barros LM, Silva LA, Caetano JA. Avaliação de tecnologia educativa sobre lesão por pressão baseada em indicadores de qualidade assistenciais. Rev Rene [Internet]. 2018 [cited 2019 Jul 25]; 29: e32695. Available from: <http://periodicos.ufc.br/rene/article/view/32695/pdf_l>.

3. VanGilder C, Lachenbruch C, Algrim-Boyle C, Meyer S. The International Pressure Ulcer Prevalence ${ }^{\text {Tm }}$ Survey: 2006-2015: A 10-Year Pressure Injury Prevalence and Demographic Trend Analysis by Care Setting. J Wound Ostomy Continence Nurs. 2017 Jan/Fev; 44(1):20-28.

4. Pieper M. Pressure ulcers: prevalence, incidence, and implications for the future. Washington, DC: National Pressure Ulcer Advisory Panel; 2013.

5. Briggs M, Collinson M, Wilson L, Rivers C, Mcginnis E, Dealey C. The prevalence of pain at pressure areas and pressure ulcers in hospitalised patients. BMC Nurs [Internet]. 2013 Jul [cited 2019 Jul 25]; 12(1):1-6. Available from: <https:// www.ncbi.nlm.nih.gov/pmc/articles/PMC3765382/pdf/1472-6955-12-19.pdf>

6. Soares CF, Heidemann ITSB. Promoção da saúde e prevenção da lesão por pressão: expectativas do enfermeiro da atenção primária. Texto Contexto Enferm [Internet]. 2018 May [cited Jul 25]; 27(2): el630016. Available from: <http://www.scielo.br/pdf/tce/v27n2/0104-0707-tce-27-02-el630016.pdf>.

7. Rabeh SAN, Gonçalves MBB, Caliri MHL, Nogueira PC, Miyazaki MY. Terapia tópica para feridas crônicas: contribuições de um módulo de ensino à distância para o conhecimento de estudantes de enfermagem. Enferm. Glob [Internet]. 2017 [cited 2019 Jul 25]. 16(45): 80-90. Available from: <http://scielo.isciii. es/pdf/eg/v16n45/pt_1695-6141-eg-16-45-00069.pdf>

8. MunizAP. Situação-problema de cliente com ferida neoplásica: Contribuições para a prática e ensino de enfermagem. Dissertação apresentada ao Programa de Mestrado Profissional em Ensino na Saúde da Escola de Enfermagem Aurora de Afonso Costa da Universidade Federal Fluminense. Niterói, 2017.

9. Carvalho V. Sobre enfermagem: ensino e perfil profissional. Rio de Janeiro: Ed. UFRJ; 2006
10. Carvalho V. Sobre a lex - art e arte da enfermagem: a (inter) dependência entre verdade, necessidade e vontade no fazer, ensinar e investigar. 1th ed. São Caetano do Sul, SP: Yedis; 2014

11. Aquilante AG, Silva RF, Avó LRS, Gonçalves FGP, Souza, MBB. Situações-problema Simuladas: uma Análise do Processo de Construção. Rev Bras Educ Méd [Internet]. 2011 Jun [cited 2019 Ago 14]: 35(2): Rio de Janeiro, v. 35 n. 2, p. 147-156, junho 2011. Disponivel em: <http://www.scielo.br/pdf/rbem/ v35n2/02.pdf>.

12. Moran JM, Massetto MT, Behrens MA. Novas tecnologias e mediações pedagógicas. Campinas, SP: Papirus; 2012

13. Almeida RGS, Mazzo A, Martins JCA, Baptista RCN, Girão FB, Mendes IAC Validação para lingua portuguesa da escala Student Satisfaction and Self-Confidence in Learning. Rer latinoam enferm [Internet]. 2015 Nov-Dez [cited 2019 Ago 14]; 23(6): 1007-13. Available from: <http://www.scielo.br/pdf/rlae/ v23n6/pt_0104-1169-rlae-23-06-01007.pdf>.

14. Klein NA, Ahlert EM. Aprendizagem baseada em problemas como metodologia ativa na educação profissional. Artigo de Especialização apresentado ao Curso de Docência na Educação Profissional da Universidade do Vale do Taquari. Lajeado, 2018.

15. Queluci GC, Figueiredo NMA. Sobre as situações de enfermagem e seus graus de complexidade - menor, média e maior - na prática assistencial hospitalar. Esc Anna Nery Rev Enferm [Internet]. 2010 [cited 2019 Ago 14]; 14(1): 171 - 177. Available from: <http://www.scielo.br/pdf/ean/vl4nl/vl4nla25.pdf >

16. Button D. Harrington A. Belan I. E-learning \& information communication technology (ICT) in nursing education: a review of the literature. Nurse Educ Today. 2014 Out; 34(10): 1311-23.

17. Cavalcante MLSN, Borges CL, Moura AMFTM, Carvalho RMFL. Indicadores de saúde e segurança entre idosos institucionalizados. Rev Esc Enferm USP [Internet]. 2016 [cited 2019 Jul 25]; 50 (4): 602-9. Available from: < http://www. scielo.br/pdf/reeusp/v50n4/pt_0080-6234-reeusp-50-04-0602.pdf>

RECEBIDO: 29/09/2019 - ACEITO: 30/01/2020 